\title{
Coopetition in quantum prisoner's dilemma and COVID-19
}

\author{
Ahmed S. Elgazzar ${ }^{1}$ (D)
}

Received: 22 September 2020 / Accepted: 24 February 2021 / Published online: 10 March 2021

(c) The Author(s), under exclusive licence to Springer Science+Business Media, LLC, part of Springer Nature 2021

\begin{abstract}
Decision-making is important especially during a crisis such as the novel COVID-19 pandemic. The quantum prisoner's dilemma with two dilemma strength parameters is introduced as a model for the interaction between pharmaceutical and other related enterprises during the pandemic. Novel Nash equilibria are identified. The coopetition equilibrium (simultaneous cooperation and competition) is emphasized. Motivated by the novel equilibria of the quantum version, a classical mixed-strategy formulation that can be applied to real-world situations is proposed. Suitable values of the dilemma strength parameters and quantum entanglement can encourage coopetition, which can be considered as a route to full cooperation.
\end{abstract}

Keywords Quantum prisoner's dilemma · Nash equilibrium · Dilemma strength parameters · Quantum entanglement · Coopetition · COVID-19

\section{Introduction}

Explaining decision-making problems in social, economic, and political systems is one of the principal objectives of game theory $[1,2]$. A game consists of a set of players and a set of strategies. Every player chooses a strategy and receives a payoff accordingly. The Nash equilibrium (NE) is an important concept in game theory. It is a strategy combination with the characteristic that no player can individually maximize his or her own payoff by deviating from it. No player can obtain a payoff higher than the Pareto optimal (PO) one without reducing the payoff of the opponent. An interesting general formulation of symmetric, two-player, two-strategy games is introduced in [3-5] by defining two types of universal scaling parameters. The prisoner's dilemma (PD) is the most well-known symmetric, two-player and two-strategy game. It is characterized by a unique NE that is not PO. This game models the dilemma between individual

Ahmed S. Elgazzar

aselgazzar@aru.edu.eg

1 Mathematics Department, Faculty of Science, Arish University, 45516 Arish, Egypt 
Table 1 Payoff matrix of the general classical PD model for the interaction of two pharmaceutical enterprises A and $\mathrm{B}$

\begin{tabular}{lll}
\hline & B: $C$ & B: $D$ \\
\hline A: $C$ & $(R, R)$ & $(S, T)$ \\
A: $D$ & $(T, S)$ & $(P, P)$ \\
\hline
\end{tabular}

and group interests, which is important in life. The PD has been used in a variety of scientific fields [6-9].

Quantum game theory [10-24] is an important extension of game theory using concepts from quantum information theory [25]. In quantum games, the strategic space is expanded, and novel NEs can be obtained. The quantum extension of game theory expands its fields of application.

The novel COVID-19 pandemic is a major global health threat [26]. It preoccupies many researchers as well as the global community [26-30]. This pandemic is characterized by the substantial role played by human decisions in transmitting or containing it [29]. When human behavior is competitive, resources are not used in a way that is most efficient for the community. Most of the literature in game theory studies individual behavior. This is particularly relevant during the current COVID-19 pandemic, when decisions are made by individuals and local governments. Do we act for the common good, or do we do what we perceive as serving our individual interests? Moreover, coopetition, simultaneous cooperation and competition occurring together, is highly expected in COVID-19 management [30]. Some examples of coopetition behavior include sharing resources among pharmaceutical enterprises to hasten the development of a vaccine. Many publishers have provided open access to scientific research related to COVID-19. The classical PD does not appear to offer anything new on this issue, but the quantum PD (QPD) might be able to help.

Our aim is to model the interaction between pharmaceutical enterprises or other relevant institutions during the COVID-19 pandemic using the QPD with two dilemma strength parameters. The remainder of this paper is organized as follows. In Sect. 2, the classical PD is reviewed briefly. Section 3 is devoted to some forms of the QPD in various strategic spaces. In Sect. 4, a quantum model is presented, and recently observed coopetition behavior is highlighted. Conclusions are drawn in Sect. 5.

\section{Classical prisoner's dilemma}

Interaction between pharmaceutical enterprises can be modeled using the classical PD [2]. Consider two enterprises A and B: each of them chooses a strategy, cooperate $(C)$, or compete $(D)$. Let $R, S, T$, and $P$ denote the reward, sucker, temptation, and punishment payoff, respectively, with $T>R>P>S$. The payoff of each player is determined according to the payoff matrix in Table 1. Even in a mixed-strategy game, the strategy combination $(D, D)$ is the unique NE that is not PO. This means that each of the enterprises competes and receives payoff $P$.

In the general formulation of two-player, two-strategy games with two dilemma strength parameters, two universal scaling parameters [3-5] are defined. The strength 
Table 2 Rescaled payoff matrix of a general two-player, two-strategy game using the universal scaling parameters

\begin{tabular}{lll}
\hline & B: $C$ & B: $D$ \\
\hline A: $C$ & $(R, R)$ & $\left(P-D_{\mathrm{r}}, R+D_{\mathrm{g}}\right)$ \\
A: $D$ & $\left(R+D_{\mathrm{g}}, P-D_{\mathrm{r}}\right)$ & $(P, P)$ \\
\hline
\end{tabular}

of the gamble-intending dilemma is $D_{\mathrm{g}}=T-R$, and the strength of the risk-averting dilemma is $D_{\mathrm{r}}=P-S$. The payoff matrix can be rescaled accordingly as shown in Table 2. Positive $D_{\mathrm{g}}$ values tempt players to exploit each other. On the other hand, if $D_{\mathrm{r}}$ is positive, then exploitation is to be avoided. When both $D_{\mathrm{g}}$ and $D_{\mathrm{r}}$ are positive, players are tempted to compete, and the game reduces to the classical PD.

Without loss of generality, we can set arbitrary values for both $R$ and $P$ satisfying the condition $R>P$. Let $R=1$ and $P=0$. The classical mixed-strategy PD is formulated by assuming that agent $\mathrm{A}(\mathrm{B})$ adopts strategy $C$ with probability $p(q)$ and adopts strategy $D$ with probability $1-p(1-q)$, where $0 \leq p, q \leq 1$. Using the payoff matrix in Table 2, the agents' expected payoff functions are given by

$$
\$_{\mathrm{A}}^{c}\left(p, q, D_{\mathrm{r}}, D_{\mathrm{g}}\right)=\left(D_{\mathrm{r}}-D_{\mathrm{g}}\right) p q-D_{\mathrm{r}} p+\left(1+D_{\mathrm{g}}\right) q
$$

and

$$
\$_{\mathrm{B}}^{c}\left(p, q, D_{\mathrm{r}}, D_{\mathrm{g}}\right)=\left(D_{\mathrm{r}}-D_{\mathrm{g}}\right) p q-D_{\mathrm{r}} q+\left(1+D_{\mathrm{g}}\right) p
$$

A probability combination $\left(p^{*}, q^{*}\right)$ is a mixed-strategy NE if

$$
\$_{\mathrm{A}}^{c}\left(p^{*}, q^{*}, D_{\mathrm{r}}, D_{\mathrm{g}}\right) \geq \$_{\mathrm{A}}^{c}\left(p, q^{*}, D_{\mathrm{r}}, D_{\mathrm{g}}\right), \forall p \in[0,1]
$$

and

$$
\$_{\mathrm{B}}^{c}\left(p^{*}, q^{*}, D_{\mathrm{r}}, D_{\mathrm{g}}\right) \geq \$_{\mathrm{B}}^{c}\left(p^{*}, q, D_{\mathrm{r}}, D_{\mathrm{g}}\right), \forall q \in[0,1]
$$

Thus, the strategy combination $(D, D)$ is the unique NE, and the dilemma still exists.

\section{Quantum prisoner's dilemma in various strategic spaces}

In the Eisert-Wilkens-Lewenstein (EWL) quantization scheme [10,11] for the PD, the state of each player is described fully by a qubit in the Hilbert space of a two-state system: either $|C\rangle=\left(\begin{array}{l}1 \\ 0\end{array}\right)$ or $|D\rangle=\left(\begin{array}{l}0 \\ 1\end{array}\right)$. The initial state of the game is assumed to be $|C C\rangle$. Then, a general entangling operator

$$
\hat{J}(\gamma)=\cos \left(\frac{\gamma}{2}\right) \hat{I} \otimes \hat{I}+\imath \sin \left(\frac{\gamma}{2}\right) \hat{\sigma_{x}} \otimes \hat{\sigma}_{x}
$$

is applied, where $\hat{I}$ is the identity operator, $\hat{\sigma}_{x}$ is the Pauli-spin flip operator, and $0 \leq \gamma \leq \frac{\pi}{2}$ is the entanglement degree. At $\gamma=0$, the initial state is separable, 
while at $\gamma=\frac{\pi}{2}$, the game is maximally entangled. Each player is allowed to choose a quantum strategy, $\hat{U}_{j}, j=\mathrm{A}, \mathrm{B}$. Then, a disentangling operator $\hat{J}^{\dagger}$ is applied, and the final state of the game becomes

$$
\left|\psi_{f}\right\rangle=\hat{J}^{\dagger} \hat{U}_{\mathrm{A}} \otimes \hat{U}_{\mathrm{B}} \hat{J}|C C\rangle
$$

The player's expected payoff functions are calculated according to

$$
\begin{aligned}
& \$_{\mathrm{A}(\mathrm{B})}=P_{\mathrm{A}(\mathrm{B})}(C, C) \Pi_{\mathrm{CC}}+P_{\mathrm{A}(\mathrm{B})}(C, D) \Pi_{\mathrm{CD}} \\
& +P_{\mathrm{A}(\mathrm{B})}(D, C) \Pi_{\mathrm{DC}}+P_{\mathrm{A}(\mathrm{B})}(D, D) \Pi_{\mathrm{DD}},
\end{aligned}
$$

where $P_{\mathrm{A}(\mathrm{B})}$ is the classical payoff of player A (B) and $\Pi_{\sigma \grave{\sigma}}=\left|\left\langle\sigma \grave{\sigma} \mid \psi_{f}\right\rangle\right|^{2}$ is the joint probability that the final state will collapse to $|\sigma \dot{\sigma}\rangle$ and $\sigma=C, D$.

Consider the strategic space to be the space of operators $\mathrm{SU}(2)$, then

$$
\hat{U}_{j}\left(\alpha_{j}, \beta_{j}\right)=\left\{\left(\begin{array}{cc}
\alpha_{j} & \beta_{j} \\
-\bar{\beta}_{j} & \bar{\alpha}_{j}
\end{array}\right), \alpha_{j}, \beta_{j} \in \mathbb{C},\left|\alpha_{j}\right|^{2}+\left|\beta_{j}\right|^{2}=1 \text {, and } j=\mathrm{A}, \mathrm{B}\right\} \text {. }
$$

This space is known to be closed under composition; hence, for each quantum strategy $\hat{U}(\alpha, \beta)$, there is a counter-strategy $\hat{U}(-\bar{\beta}, \bar{\alpha})$. Therefore, there is no NE for the quantum game under this general strategic space, in agreement with $[19,20]$.

Several subsets of the space $S U(2)$ have been proposed as strategic spaces. Eisert et al. [10] defined the two-parameter strategic space in (9),

$$
\hat{U}_{j}\left(\theta_{j}, \phi_{j}\right)=\left\{\left(\begin{array}{cc}
e^{l \phi_{j}} \cos \left(\theta_{j} / 2\right) & \sin \left(\theta_{j} / 2\right) \\
-\sin \left(\theta_{j} / 2\right) & e^{-l \phi_{j}} \cos \left(\theta_{j} / 2\right)
\end{array}\right): \theta_{j} \in[0, \pi], \phi_{j} \in\left[0, \frac{\pi}{2}\right] \text {, and } j=\mathrm{A}, \mathrm{B}\right\} \text {. }
$$

Also, they defined the quantum strategies: $\hat{D} \equiv \hat{U}(\pi, 0)=\left(\begin{array}{cc}0 & 1 \\ -1 & 0\end{array}\right)$ and $\hat{Q} \equiv$ $\hat{U}\left(0, \frac{\pi}{2}\right)=\left(\begin{array}{cc}l & 0 \\ 0 & -l\end{array}\right)$ for compete and quantum cooperate, respectively. Novel features are observed, where the NE properties are found to depend on the entanglement parameter $\gamma[10,11]$. The alternative two-parameter strategic space in (10) was defined in $[20,21]$.

$$
\hat{U}_{j}\left(\theta_{j}, \phi_{j}\right)=\left\{\left(\begin{array}{cc}
\cos \left(\theta_{j} / 2\right) & \imath e^{\imath \phi_{j}} \sin \left(\theta_{j} / 2\right) \\
\iota e^{-l \phi_{j}} \sin \left(\theta_{j} / 2\right) & \cos \left(\theta_{j} / 2\right)
\end{array}\right): \theta_{j} \in[0, \pi], \phi_{j} \in\left[0, \frac{\pi}{2}\right], \text { and } j=\mathrm{A}, \mathrm{B}\right\}
$$

Different NE properties were obtained.

Eisert and Wilkens [22] supposed a restricted one-parameter strategic space in the form described in (11).

$$
\hat{U}_{j}\left(\theta_{j}\right)=\left\{\left(\begin{array}{cc}
\cos \left(\theta_{j} / 2\right) & \sin \left(\theta_{j} / 2\right) \\
-\sin \left(\theta_{j} / 2\right) & \cos \left(\theta_{j} / 2\right)
\end{array}\right): \theta_{j} \in[0, \pi], \text { and } j=\mathrm{A}, \mathrm{B}\right\}
$$


The game was reduced to the classical mixed-strategy PD. Elgazzar [17] introduced the simple one-parameter strategic space in (12) and (13) as a superposition of the quantum strategies $\hat{Q}$ and $\hat{D}$.

$$
\begin{aligned}
& \hat{U}_{\mathrm{A}}(p)=\left\{\left(\begin{array}{cc}
l \sqrt{p} & \sqrt{1-p} \\
-\sqrt{1-p} & -l \sqrt{p}
\end{array}\right), 0 \leq p \leq 1\right\} \\
& \hat{U}_{\mathrm{B}}(q)=\left\{\left(\begin{array}{cc}
l \sqrt{q} & \sqrt{1-q} \\
-\sqrt{1-q} & -l \sqrt{q}
\end{array}\right), 0 \leq q \leq 1\right\}
\end{aligned}
$$

The agent A (B) coopetates $(\hat{Q})$ with probability $p(q)$ and competes $\hat{D}$ with probability $1-p(1-q)$. This strategic space preserves the novel features of the EWL scheme and improves its NE properties in addition to simplifying calculations. For this reason, we use it in our quantum model in the next section.

Studying NEs in quantum games is important. Based on the EWL scheme with a maximally entangled quantum initial state, Landsburg [23] has classified all potential NEs for generic two-player, two-strategy games. Ahmed [24] has generalized Landsburg's classification to an arbitrary maximally entangled quantum initial state $\frac{1}{\sqrt{2}}\left(|C C\rangle+e^{i \theta}|D D\rangle\right)$, where $\theta$ is a real number.

\section{Quantum model}

Here, we present a quantum model based on the quantization scheme in [17]. Using the strategic space defined by (12) and (13), and the classical payoff matrix (Table 2) with $R=1$ and $P=0$, the expected payoff functions are given by

$$
\$_{\mathrm{A}}^{q}=\Pi_{\mathrm{CC}}-D_{r} \Pi_{\mathrm{CD}}+\left(1+D_{g}\right) \Pi_{\mathrm{DC}}
$$

and

$$
\$_{\mathrm{B}}^{q}=\Pi_{\mathrm{CC}}+\left(1+D_{\mathrm{g}}\right) \Pi_{\mathrm{CD}}-D_{\mathrm{r}} \Pi_{\mathrm{DC}}
$$

This yields

$$
\begin{aligned}
& \$_{A}^{q}\left(p, q, D_{\mathrm{g}}, D_{\mathrm{r}}, \gamma\right) \\
& \quad=\left(D_{\mathrm{r}}-D_{\mathrm{g}}\right) p q-D_{\mathrm{r}} p+\left(1+D_{\mathrm{g}}\right) q+\left(1+D_{\mathrm{r}}+D_{\mathrm{g}}\right)(p-q) \sin ^{2} \gamma
\end{aligned}
$$

and

$$
\begin{aligned}
& \$_{B}^{q}\left(p, q, D_{\mathrm{g}}, D_{\mathrm{r}}, \gamma\right)= \\
& \quad\left(D_{\mathrm{r}}-D_{\mathrm{g}}\right) p q-D_{\mathrm{r}} q+\left(1+D_{\mathrm{g}}\right) p-\left(1+D_{\mathrm{r}}+D_{\mathrm{g}}\right)(p-q) \sin ^{2} \gamma .
\end{aligned}
$$


Table 3 Possible NE domains of the quantum model and the corresponding payoffs. In this table, $\gamma_{1}=$ $\arcsin \left(\sqrt{D_{\mathrm{r}} /\left(1+2 D_{\mathrm{r}}+\Delta\right)}\right), \gamma_{2}=\arcsin \left(\sqrt{\left(D_{\mathrm{r}}+\Delta\right) /\left(1+2 D_{\mathrm{r}}+\Delta\right)}\right), \pi_{D}=1+D_{\mathrm{r}}+\Delta-\left(1+2 D_{\mathrm{r}}+\Delta\right.$ $\sin ^{2} \gamma, \pi_{Q}=-D_{\mathrm{r}}+\left(1+2 D_{\mathrm{r}}+\Delta\right) \sin ^{2} \gamma, p^{*}=q^{*}=\left[\left(1+2 D_{\mathrm{r}}+\Delta\right) \sin ^{2} \gamma-D_{\mathrm{r}}\right] / \Delta, \hat{Q}^{*}=\hat{U}\left(p^{*}\right)$, and $\pi^{*}=-\Delta p^{* 2}+(1+\Delta) p^{*}$

\begin{tabular}{lccc}
\hline Dilemma strength parameters & Entanglement & NE & Payoff \\
\hline & $0 \leq \gamma \leq \gamma_{1}$ & $\hat{D} \otimes \hat{D}$ & $(0,0)$ \\
& $\gamma_{1} \leq \gamma \leq \gamma_{2}$ & $\hat{D} \otimes \hat{Q}$ & $\left(\pi_{D}, \pi_{Q}\right)$ \\
$D_{\mathrm{g}}>D_{\mathrm{r}}$ & $\hat{Q}^{*} \otimes \hat{Q}^{*}$ & $\left(\pi^{*}, \pi^{*}\right)$ \\
& $\gamma_{2} \leq \gamma \leq \frac{\pi}{2}$ & $\hat{Q} \otimes \hat{D}$ & $\left(\pi_{Q}, \pi_{D}\right)$ \\
& $0 \leq \gamma \leq \gamma_{1}$ & $\hat{Q} \otimes \hat{Q}$ & $(1,1)$ \\
\hline$D_{\mathrm{g}}=D_{\mathrm{r}}$ & $\gamma_{1} \leq \gamma \leq \frac{\pi}{2}$ & $\hat{Q} \otimes \hat{D}$ & $(0,0)$ \\
& $0 \leq \gamma \leq \gamma_{2}$ & $\hat{D} \otimes \hat{Q}$ & $(1,1)$ \\
\hline & $\gamma_{2} \leq \gamma \leq \gamma_{1}$ & $\hat{D} \otimes \hat{D}$ & $(0,0)$ \\
$D_{\mathrm{g}}<D_{\mathrm{r}}$ & $\gamma_{1} \leq \gamma \leq \frac{\pi}{2}$ & $\hat{Q} \otimes \hat{Q}$ & $(0,0)$ \\
& & & $(1,1)$ \\
& & &
\end{tabular}

A strategy product $\hat{U}_{\mathrm{A}}\left(p^{*}\right) \otimes \hat{U}_{\mathrm{B}}\left(q^{*}\right)$ is an NE if the corresponding probability combination $\left(p^{*}, q^{*}\right)$ satisfies the conditions (18) and (19).

$$
\begin{aligned}
& \$_{\mathrm{A}}^{q}\left(p^{*}, q^{*}, D_{\mathrm{g}}, D_{\mathrm{r}}, \gamma\right)-\$_{\mathrm{A}}^{q}\left(p, q^{*}, D_{\mathrm{g}}, D_{\mathrm{r}}, \gamma\right) \geq 0, \quad \forall \quad p \in[0,1] \\
& \$_{\mathrm{B}}^{q}\left(p^{*}, q^{*}, D_{\mathrm{g}}, D_{\mathrm{r}}, \gamma\right)-\$_{\mathrm{B}}^{q}\left(p^{*}, q, D_{\mathrm{g}}, D_{\mathrm{r}}, \gamma\right) \geq 0, \quad \forall \quad q \in[0,1]
\end{aligned}
$$

This yields (20) and (21).

$$
\begin{aligned}
& \left(p-p^{*}\right)\left[\left(D_{\mathrm{g}}-D_{\mathrm{r}}\right) q^{*}+D_{\mathrm{r}}-\left(1+D_{\mathrm{r}}+D_{\mathrm{g}}\right) \sin ^{2} \gamma\right] \geq 0 \\
& \left(q-q^{*}\right)\left[\left(D_{\mathrm{g}}-D_{\mathrm{r}}\right) p^{*}+D_{\mathrm{r}}-\left(1+D_{\mathrm{r}}+D_{\mathrm{g}}\right) \sin ^{2} \gamma\right] \geq 0
\end{aligned}
$$

The possible NE domains are obtained, as shown in Table 3. The NE properties depend on both $\Delta=D_{\mathrm{g}}-D_{\mathrm{r}}$ and $\gamma$. Therefore, different values of the dilemma strength parameters $D_{\mathrm{g}}$ and $D_{\mathrm{r}}$ that do not affect the NE properties of the classical PD are shown to affect the NE properties of the QPD.

When $\triangle>0$, as $\gamma$ exceeds the first threshold $\gamma_{1}$, a novel symmetric NE $\hat{Q}^{*} \otimes \hat{Q}^{*}$ is obtained. This is more efficient than the two asymmetric NEs $\hat{D} \otimes \hat{Q}$ and $\hat{Q} \otimes \hat{D}$, as shown in Fig. 1. Each player obtains an increasing expected payoff from zero to one; see Fig. 1. This NE was obtained first in [17] and appears only when $D_{\mathrm{g}}>D_{\mathrm{r}}$. The NE $\hat{Q}^{*} \otimes \hat{Q}^{*}$ corresponds to coopetition (simultaneous cooperation with probability $p^{*}$ and competition with probability $1-p^{*}$ ). This coopetition behavior has been observed during the COVID-19 pandemic [23]. We present an interpretation. Sensing danger increases the temptation to act selfishly, with the result that $D_{\mathrm{g}}$ exceeds $D_{\mathrm{r}}$. At the same time, the possibility of transmission of the infection to different locations around the world increases the entanglement. Thus, the coopetition behavior appears.

As shown in Table 3 , at $\Delta=0$ the transitional interval disappears, with the result that only two domains for NE are obtained. For $\Delta<0$, the transitional interval is replaced 


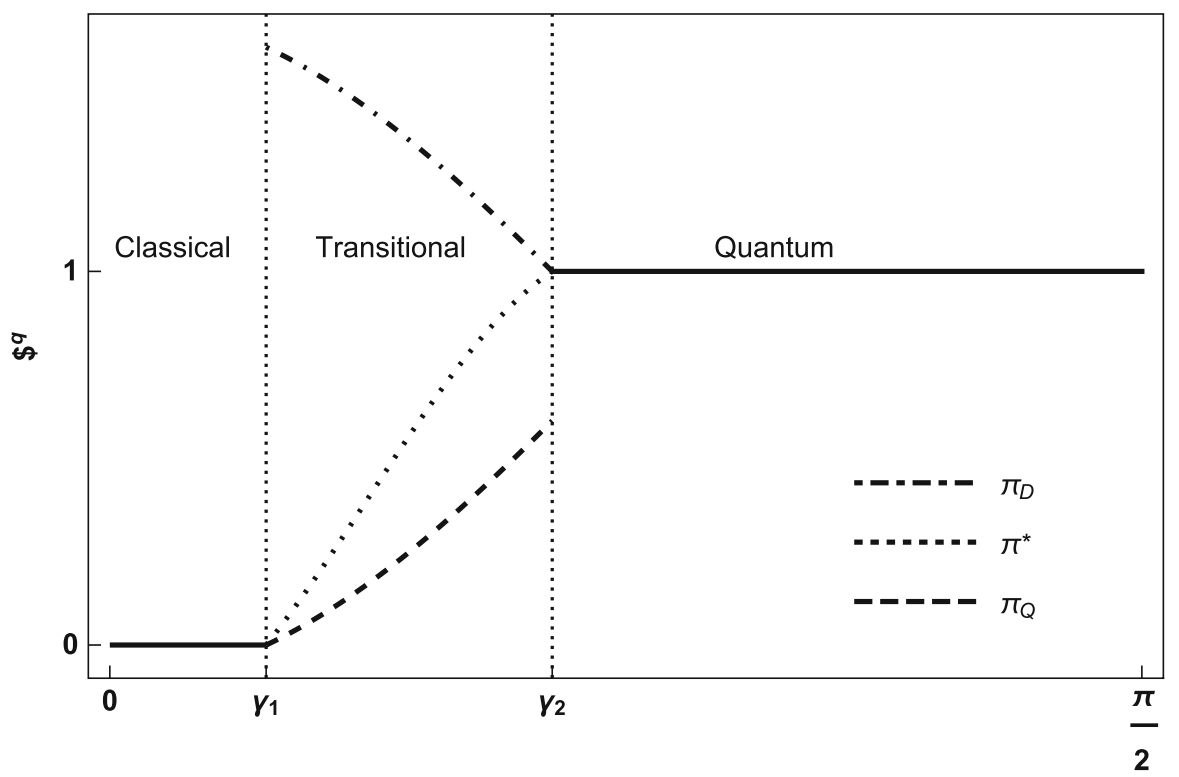

$r$

Fig. 1 Nash equilibrium expected payoff of the quantum model, $\$^{q}$, as a function of the entanglement parameter, $\gamma$. We set $D_{\mathrm{r}}=0.1$ and $D_{\mathrm{g}}=0.7$. For $0 \leq \gamma \leq \gamma_{1}, \$^{q}=0$ (competing phase). When $\gamma_{1} \leq \gamma \leq \gamma_{2}, \$^{q}=\pi^{*}$ (coopetiting phase). For $\gamma_{2} \leq \gamma \leq \frac{\pi}{2}, \$^{q}=1$ (cooperating phase). As $\gamma$ increases, the quantum model transfers continuously from competing to coopetiting, then to cooperating phases corresponding to the classical, transitional, and quantum domains, respectively

by a coexistence interval where both $\hat{D} \otimes \hat{D}$ and $\hat{Q} \otimes \hat{Q}$ are possible NEs. When $\gamma=\frac{\pi}{2}$, the initial state is maximally entangled, and the NE is $\hat{Q} \otimes \hat{Q}$ independently of $\triangle$. This NE is a pure strategy in agreement with Landsburg's classification [23].

In the following, we highlight many properties of the coopetition $\mathrm{NE}\left(\hat{Q}^{*} \otimes \hat{Q}^{*}\right)$. At $\gamma=\gamma_{1}$, the probability of cooperation, $p^{*}$ in $\hat{Q}^{*}$ is equal to zero, and the payoff of each player is equal to zero. As the entanglement increases, $p^{*}$ increases, and the payoff increases accordingly. In the middle of the transitional region, both probabilities of cooperation and competition are equal. In this case, the player's payoff is equal to $\frac{1}{4}(2+\Delta)$, which is the mean of the classical payoff matrix (Table 2). This behavior was observed previously in some limits of various QPD schemes [13-17]. At the end of the transitional region, $p^{*}=1$ (full cooperation), and each player receives a payoff one.

The length of the transitional interval is defined as $L=\gamma_{2}-\gamma_{1}$. We depict $L$ as a function of both $D_{\mathrm{r}}$ and $\triangle$ in Fig. 2. Without loss of generality, we assume that $0<D_{\mathrm{r}} \leq 1$ and $0<\Delta \leq 1$. The length $L$ increases with either an increase in $\Delta$ or a decrease in $D_{\mathrm{r}}$, especially for very small values of $D_{r}$. As $\Delta$ increases, the increase in $L$ with the decrease in $D_{\mathrm{r}}$ occurs faster.

For a deep understanding of how the entanglement parameter $\gamma$ affects the expected payoff, $\pi^{*}$, of the coopetition NE, we set $D_{\mathrm{r}}=0.1$ and explore $\pi^{*}$ as a function of $\gamma$ for different values of the parameter $\Delta$, as shown in Figure 3. In all cases, the expected 


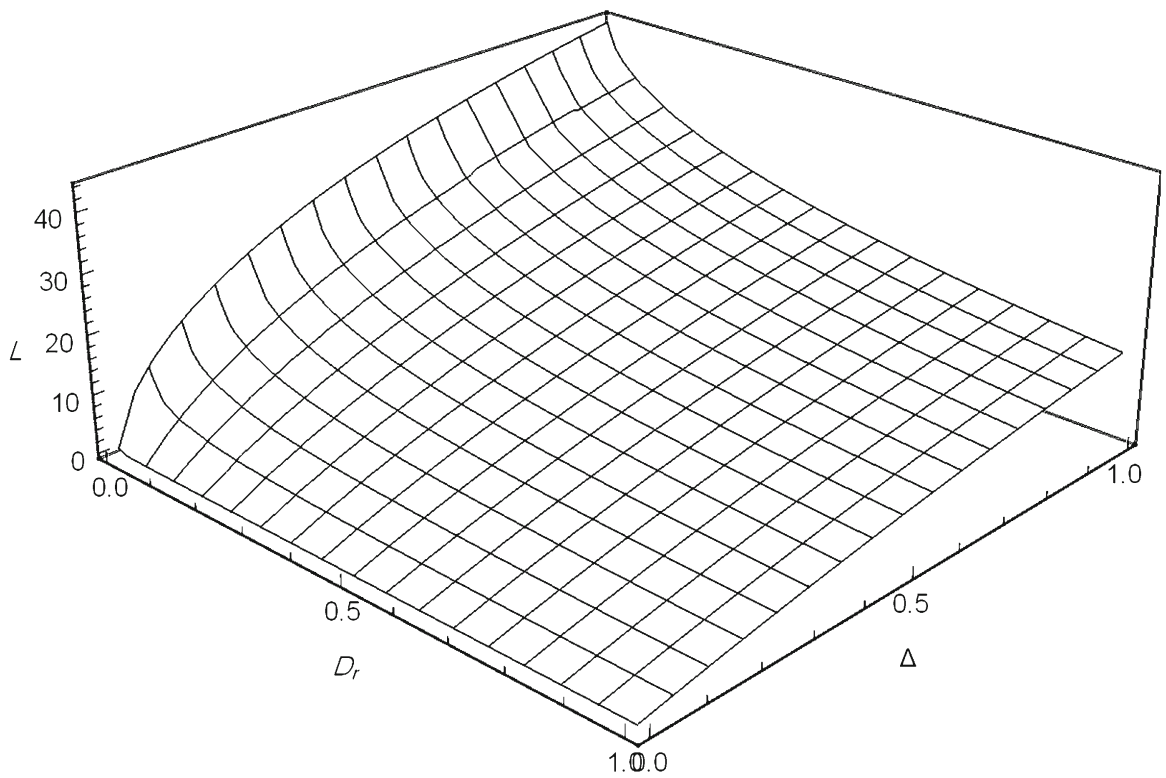

Fig. 2 Length of the transitional interval as a function of both $D_{\mathrm{r}}$ and $\Delta$. The transitional region expands with an increase in $\triangle$ and a decrease in $D_{\mathrm{r}}$, especially for small values of $D_{\mathrm{r}}$

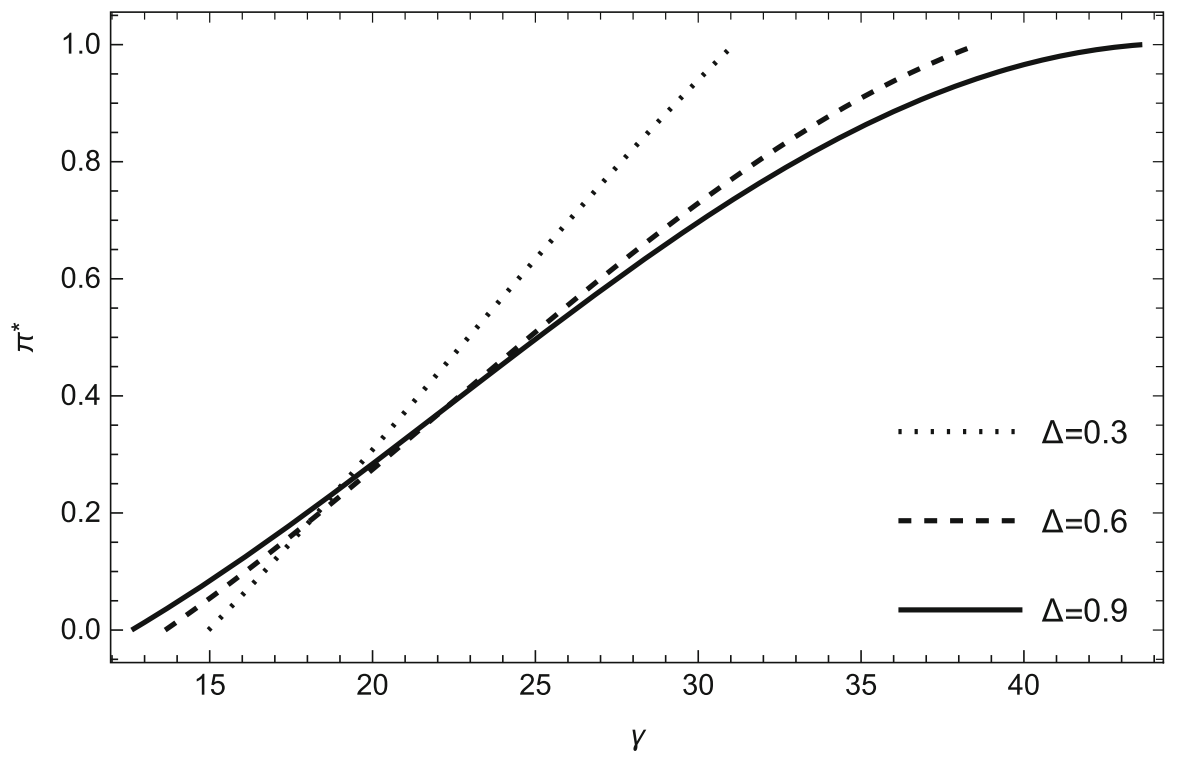

Fig. 3 Nash equilibrium expected payoff within the transitional region, $\pi^{*}$, versus the entanglement parameter, $\gamma$, for $D_{\mathrm{r}}=0.1$, and different values of $\Delta$. As $\gamma$ increases, $\pi^{*}$ increases from 0 to 1 . As $\Delta$ increases, the transitional region expands, and the increase in $\pi^{*}$ occurs more slowly 
payoff $\pi^{*}$ increases with the increase in $\gamma$ in agreement with [17], but this increase occurs more slowly as $\Delta$ increases, as a result of the expansion of the transitional region as $\triangle$ increases.

To investigate a pure cooperator-competitor conflict in our model, consider a case in which A always cooperates $(p=1)$, while B always competes $(q=0)$. From (16) and (17), the expected payoff functions become

$$
\$_{\mathrm{A}}^{q}\left(1,0, D_{\mathrm{g}}, D_{\mathrm{r}}, \gamma\right)=-D_{\mathrm{r}}+\left(1+2 D_{\mathrm{r}}+\Delta\right) \sin ^{2} \gamma
$$

and

$$
\$_{\mathrm{B}}^{q}\left(1,0, D_{\mathrm{g}}, D_{\mathrm{r}}, \gamma\right)=1+D_{\mathrm{r}}+\Delta-\left(1+2 D_{\mathrm{r}}+\Delta\right) \sin ^{2} \gamma
$$

The expected payoff function $\$_{\mathrm{A}}^{q}\left(1,0, D_{\mathrm{g}}, D_{\mathrm{r}}, \gamma\right)$ is an increasing function of $\gamma$, while $\$_{\mathrm{B}}^{q}\left(1,0, D_{\mathrm{g}}, D_{\mathrm{r}}, \gamma\right)$ is decreasing. For $\gamma<\pi / 4$, competing is more profitable. When $\gamma>\pi / 4$, cooperation is more profitable, and a cooperator can beat a competitor. Then, increasing the entanglement enhances the payoff of a cooperator against a defector in agreement with [17].

Within the transitional interval, a coopetitor-cooperator conflict results in the expected payoff functions being (24) and (25),

$$
\begin{aligned}
& \$_{\mathrm{A}}^{q}\left(p^{*}, 1, D_{\mathrm{g}}, D_{\mathrm{r}}, \gamma\right)=1+\left(p^{*}-1\right)\left[\left(1+D_{\mathrm{r}}+D_{\mathrm{g}}\right) \sin ^{2} \gamma-D_{\mathrm{g}}\right] \\
& \$_{\mathrm{B}}^{q}\left(p^{*}, 1, D_{\mathrm{g}}, D_{\mathrm{r}}, \gamma\right)=p^{*}-\left(p^{*}-1\right)\left[\left(1+D_{\mathrm{r}}+D_{\mathrm{g}}\right) \sin ^{2} \gamma-D_{\mathrm{r}}\right]
\end{aligned}
$$

with the result that $\$_{\mathrm{A}}^{q}\left(p^{*}, 1, D_{\mathrm{g}}, D_{\mathrm{r}}, \gamma\right) \geq \$_{\mathrm{B}}^{q}\left(p^{*}, 1, D_{\mathrm{g}}, D_{\mathrm{r}}, \gamma\right) \forall \gamma \in\left[\gamma_{1}, \gamma_{2}\right]$. The expected payoff functions for a coopetitor-competitor conflict are

$$
\$_{\mathrm{A}}^{q}\left(p^{*}, 0, D_{\mathrm{g}}, D_{\mathrm{r}}, \gamma\right)=p^{*}\left[\left(1+D_{\mathrm{r}}+D_{\mathrm{g}}\right) \sin ^{2} \gamma-D_{\mathrm{r}}\right]
$$

and

$$
\$_{\mathrm{B}}^{q}\left(p^{*}, 0, D_{\mathrm{g}}, D_{\mathrm{r}}, \gamma\right)=p^{*}\left[-\left(1+D_{\mathrm{r}}+D_{\mathrm{g}}\right) \sin ^{2} \gamma+1+D_{\mathrm{g}}\right]
$$

Then, $\$_{\mathrm{A}}^{q}\left(p^{*}, 0, D_{\mathrm{g}}, D_{\mathrm{r}}, \gamma\right)>\$_{\mathrm{B}}^{q}\left(p^{*}, 0, D_{\mathrm{g}}, D_{\mathrm{r}}, \gamma\right)$ for $\gamma>\pi / 4$, and vice versa. Therefore, the coopetition strategy $\hat{Q}^{*}$ is more efficient than both the cooperation strategy $\hat{Q}$ and the competition strategy $\hat{D}$ within the transitional interval.

The impact of the environment on quantum systems is very important. It can be modeled by considering corrupted sources for the initial state $[12,13,17]$. The corrupted sources produce the state $|D\rangle$ with a corruption rate $0 \leq r \leq 1$ and the state $|C\rangle$ with probability $1-r$. The competition NE, $\hat{D} \otimes \hat{D}$ is robust, while the cooperation NE, $\hat{Q} \otimes \hat{Q}$ is not robust under corruption [17]. Here, we are interested in investigating the robustness of the coopetition NE, $\hat{Q}^{*} \otimes \hat{Q}^{*}$. Based on [17], the degree of robustness, 


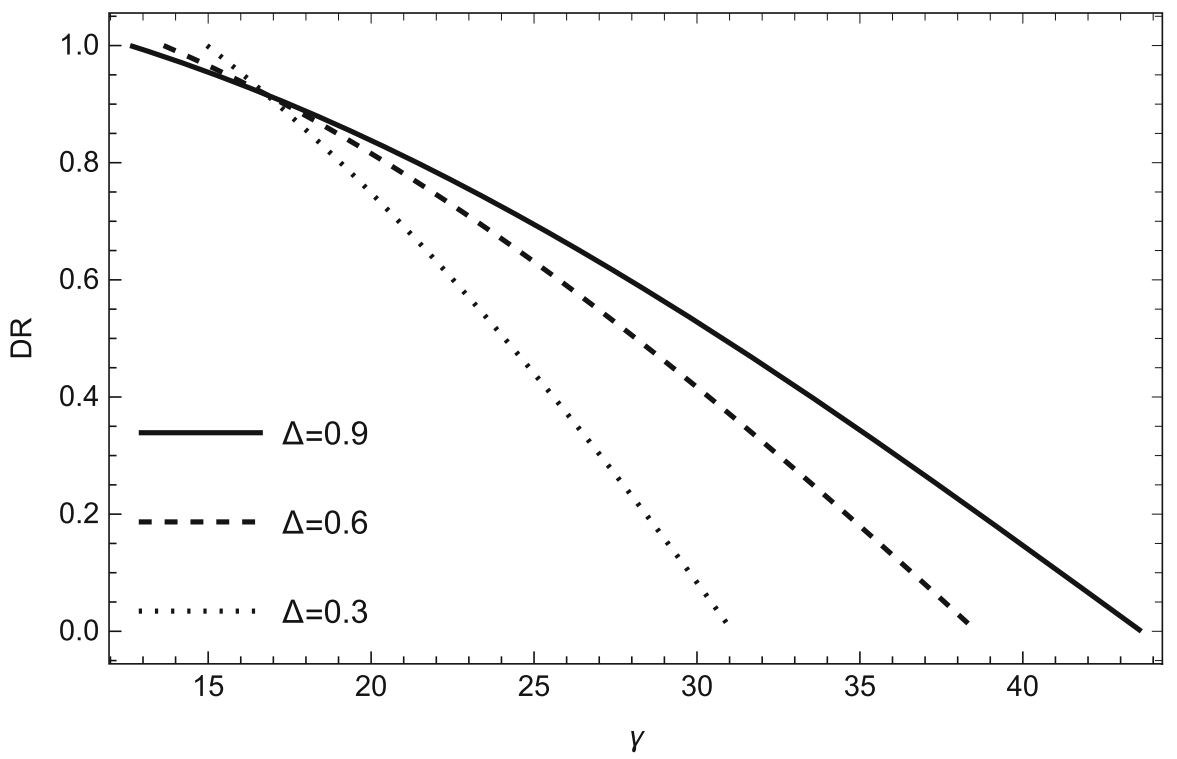

Fig. 4 Degree of robustness, DR, of the Nash equilibrium within the transitional region as a function of the entanglement parameter, $\gamma$, for $D_{\mathrm{r}}=0.1$, and different values of $\Delta$. As $\gamma$ increases, DR decreases. As $\Delta$ increases, the transitional region expands, and the reduction of DR occurs more slowly

$0 \leq D R \leq 1$, of the NE of the QPD with respect to corruption of the source is defined as in (28).

$$
\operatorname{DR}\left(D_{\mathrm{g}}, D_{\mathrm{r}}, \gamma\right)= \begin{cases}1, & 0 \leq \gamma \leq \gamma_{1} \\ \frac{1}{D_{\mathrm{g}}-D_{\mathrm{r}}}\left[D_{\mathrm{g}}-\left(1+D_{\mathrm{r}}+D_{\mathrm{g}}\right) \sin ^{2} \gamma\right], & \gamma_{1} \leq \gamma \leq \gamma_{2} \\ 0, & \gamma_{2} \leq \gamma \leq \frac{\pi}{2}\end{cases}
$$

In the transitional interval, the degree of robustness of the coopetition NE can be expressed as (29).

$$
\operatorname{DR}\left(D_{\mathrm{r}}, \Delta, \gamma\right)=\frac{1}{\Delta}\left[D_{\mathrm{r}}+\Delta-\left(1+2 D_{\mathrm{r}}+\Delta\right) \sin ^{2} \gamma\right]
$$

Figure 4 shows DR as a function of $\gamma$ for different values of $\Delta$ at $D_{\mathrm{r}}=0.1$. As previously observed in $[12,13,17]$, as the entanglement increases, the robustness of NE (DR) decreases and the system become more sensitive to mistakes. Increasing $\triangle$ slows the rate of DR reduction with increasing $\gamma$. Therefore, mistakes are to be reduced when dealing with such entangled systems. Entanglement is to be limited to the minimal value, which allows the emergence of the coopetition behavior, when mistakes are expected.

The expected payoff functions of the quantum model (16) and (17) can be expressed in terms of its analogs in the classical mixed-strategy model (1) and (2) as

$$
\$_{\mathrm{A}}^{q}=\$_{\mathrm{A}}^{c} \cos ^{2} \gamma+\$_{\mathrm{B}}^{c} \sin ^{2} \gamma
$$


and

$$
\$_{\mathrm{B}}^{q}=\$_{\mathrm{B}}^{c} \cos ^{2} \gamma+\$_{\mathrm{A}}^{c} \sin ^{2} \gamma
$$

Then, the global classical payoff $\left(\$_{\mathrm{A}}^{c}+\$_{\mathrm{B}}^{c}\right)$ is preserved in the quantum model. The effect of quantization is only in sharing the payoffs according to (30) and (31). Applying the condition that the payoffs are shared according to (30) and (31) to the classical mixed-strategy PD yields the expected payoff functions of the quantum model (16) and (17), with all NEs in Table (3) being identified. In this situation, $p(q)$ are the classical probability of cooperation of player A (B). With this formulation, our results become applicable to real-world scenarios.

\section{Conclusion}

In the COVID-19 pandemic, full cooperation or at least coopetition is necessary for controlling the infection and for sustainability. The NE properties of the quantum model are shown to depend on the dilemma strength parameters and the quantum entanglement. Then, values of the dilemma strength parameters that do not affect the NE properties of the classical PD are shown to influence the NE properties of the QPD significantly. When $D_{\mathrm{g}}>D_{\mathrm{r}}$, there are three regions for NE: classical (competition), transitional (coopetition), and quantum (cooperation), according to the entanglement level. Within the transitional interval, coopetition is more efficient than both cooperation and competition. Quantum entanglement is found to enhance the payoff of the coopetition NE, but it reduces the robustness of the coopetition NE with respect to corruption of the sources. The increase of the difference $\Delta=D_{\mathrm{g}}-D_{\mathrm{r}}$ slows these processes. At the middle of the transitional interval, the payoff of the coopetition NE is equal to the average of the classical payoff matrix. The transitional interval expands with an increase in $\Delta$. It can cover the entire range $[0, \pi / 2]$ for very large values of $\Delta$, thereby delaying attainment of the full cooperation NE. Hence, differences in $\Delta$ are not to be overrated. In addition, mistakes are to be avoided, because increasing the entanglement leads to increased sensitivity to mistakes and environmental influences.

Acknowledgements I am very grateful to the reviewers for the constructive comments and suggestions.

\section{References}

1. von Neumann, J., Morgenstern, O.: Theory of games and economic behavior. Princeton University Press, New Jersey (1944)

2. Colman, A.M.: Game theory and its applications in the social and biological sciences. ButterworthHeinemann, Oxford (1995)

3. Tanimoto, J., Sagara, H.: Relationship between dilemma occurrence and the existence of a weakly dominant strategy in a two-player symmetric game. Biosystems 90, 105-114 (2007). https://doi.org/ 10.1016/j.biosystems.2006.07.005

4. Wang, Z., Kokubo, S., Jusup, M., Tanimoto, J.: Universal scaling for the dilemma strength in evolutionary games. Phys. Life Rev. 14, 1-30 (2015). https://doi.org/10.1016/j.plrev.2015.04.033 
5. Ito, H., Tanimoto, J.: Scaling the phase-planes of social dilemma strengths shows game-class changes in the five rules governing the evolution of cooperation. R. Soc. Open Sci. 5, 181085 (2018). https:// doi.org/10.1098/rsos.181085

6. Brosig, J.: Identifying cooperative behavior: some experimental results in a prisoner's dilemma game. J. Econ. Behav. Organiz. 47, 275-290 (2002). https://doi.org/10.1016/S0167-2681(01)00211-6

7. Doebeli, M., Hauert, C.: Models of cooperation based on the prisoner's dilemma and the snowdrift game. Ecol. Lett. 8, 748-766 (2005). https://doi.org/10.1111/j.1461-0248.2005.00773.x

8. Ahmed, E., Hegazi, A.S., Elgazzar, A.S.: On some variants of prisoner's dilemma dynamics. Appl. Math. Comput. 163, 163-168 (2005). https://doi.org/10.1016/j.amc.2004.01.025

9. Pothos, E.M., Perry, G., Corr, P.J., Matthew, M.R., Busemeyer, J.R.: Understanding cooperation in the prisoner's dilemma game. Pers. Individ. Differ. 51, 210-215 (2011). https://doi.org/10.1016/j.paid. 2010.05.002

10. Eisert, J., Wilkens, M., Lewenstein, M.: Quantum games and quantum strategies. Phys. Rev. Lett. 83, 3077 (1999). https://doi.org/10.1103/PhysRevLett.83.3077

11. Du, J., Xu, X., Li, H., Zhou, X., Han, R.: Entanglement playing a dominating role in quantum games. Phys. Lett. A 289, 9-15 (2001). https://doi.org/10.1016/S0375-9601(01)00575-8

12. Johnson, N.F.: Playing a quantum game with a corrupted source. Phys. Rev. A 63, 020302 (2001). https://doi.org/10.1103/PhysRevA.63.020302

13. Özdemir, ŞK., Shimamura, J., Imoto, N.: Quantum advantage does not survive in the presence of a corrupt source: optimal strategies in simultaneous move games. Phys. Lett. A 325, 104-111 (2004). https://doi.org/10.1016/j.physleta.2004.03.042

14. Chen, L.K., Ang, H., Kiang, D., Kwek, L.C., Lo, C.F.: Quantum prisoner dilemma under decoherence. Phys. Lett. A 316, 317-323 (2003). https://doi.org/10.1016/S0375-9601(03)01175-7

15. Flitney, A.P., Abbott, D.: Quantum games with decoherence. J. Phys. A: Math. Gen. 38, 449-460 (2005). https://doi.org/10.1088/0305-4470/38/2/011

16. Elgazzar, A.S.: Unique solution to the quantum prisoner's dilemma game. J. Phys. Soc. Jpn. 88, 034801 (2019). https://doi.org/10.7566/JPSJ.88.034801

17. Elgazzar, A.S.: Quantum prisoner's dilemma in a restricted one-parameter strategic space. Appl. Math. Comput. 370, 124927 (2020). https://doi.org/10.1016/j.amc.2019.124927

18. Khan, F.S., Solmeyer, N., Balu, R., Humble, T.S.: Quantum games: a review of the history, current state, and interpretation. Quant. Inf. Process. 17, 309 (2018). https://doi.org/10.1007/s11128-018-2082-8

19. Benjamin, S.C., Hayden, P.M.: Comment on Quantum games and quantum strategies. Phys. Rev. Lett. 87, 069801 (2001). https://doi.org/10.1103/PhysRevLett.87.069801

20. Flitney, A.P., Hollenberg, L.C.L.: Nash equilibria in quantum games with generalized two-parameter strategies. Phys. Lett. A 363, 381-388 (2007). https://doi.org/10.1016/j.physleta.2006.11.044

21. Du, J., Li, H., Xu, X., Shi, M., Zhou, X.: Entanglement enhanced multiplayer quantum games. Phys. Lett. A 302, 229-233 (2002). https://doi.org/10.1016/S0375-9601(02)01144-1

22. Eisert, J., Wilkens, M.: Quantum games. J. Mod. Optics 47, 2543-2556 (2000). https://doi.org/10. 1080/09500340008232180

23. Landsburg, S.E.: Nash equilibria in quantum games. Proc. Amer. Math. Soc. 139, 4423-4434 (2011)

24. Ahmed Quaternions, A.O.: arbitrary maximally entangled states, and the quantization of two player, two strategy games, Proceedings to the 14th World Multi-Conference on Systemics, Cybernetics and Informatics, Vol. I, (2010) 376-380. Available at http://www.iiis.org/CDs2010/CD2010SCI/SCI_ 2010/PapersPdf/SA281BF.pdf

25. Nielsen, M.A., Chuang, I.L.: Quantum computation and quantum information. Cambridge University Press, Cambridge (2000)

26. World Health Organization. Novel coronavirus (2019-nCoV) situation reports (2020), https://www. who.int/emergencies/diseases/novel-coronavirus-2019/situation-reports

27. Wang, C., Horby, P.W., Hayden, F.G., Gao, G.F.: A novel coronavirus outbreak of global health concern. Lancet 395, 470-473 (2020). https://doi.org/10.1016/S0140-6736(20)30185-9

28. Wang, L., Wang, Y., Chen, Y., Qin, Q.: Unique epidemiological and clinical features of the emerging: novel coronavirus pneumonia (covid-19) implicate special control measures. J. Med. Virol. 92(2020), 568-576 (2019). https://doi.org/10.1002/jmv.25748

29. Elgazzar, A.S.: Simple mathematical models for controlling COVID-19 transmission through social distancing and community awareness, arXiv:2012.13361 (2020) 
30. Crick, J.M., Crick, D.: Coopetition and COVID-19: collaborative business-to-business marketing strategies in a pandemic crisis. Ind. Market. Manag. 88, 206-213 (2020). https://doi.org/10.1016/j. indmarman.2020.05.016

Publisher's Note Springer Nature remains neutral with regard to jurisdictional claims in published maps and institutional affiliations. 